The efficiency of devices intended to reduce microfibre release during clothes washing

\title{
Napper, IE
}

http://hdl.handle.net/10026.1/16765

\subsection{6/j.scitotenv.2020.140412}

Science of The Total Environment

Elsevier BV

All content in PEARL is protected by copyright law. Author manuscripts are made available in accordance with publisher policies. Please cite only the published version using the details provided on the item record or document. In the absence of an open licence (e.g. Creative Commons), permissions for further reuse of content should be sought from the publisher or author. 
Disclaimer

'This is a near final copy of the accepted paper as submitted for publication. Readers are advised to refer to the final version of the paper which can be found at

https://www.sciencedirect.com/science/article/abs/pii/S0048969720339346?via\%3Di

$\underline{\text { hub }}$

The efficiency of devices intended to reduce microfibre release during clothes washing

\section{Abstract:}

The washing of synthetic clothes is considered to be a substantial source of microplastic to the environment. Therefore, various devices have been designed to capture microfibres released from clothing during the washing cycle. In this study, we compared 6 different devices which varied from prototypes to commercially available products. These were designed to either be placed inside the drum during the washing cycle or fitted externally to filter the effluent wastewater discharge. The aim of this study was to examine the efficacy of these devices at mitigating microfibre release from clothing during washing or capturing any microfibres released in the effluent. When compared to the amount of microfibres entering the wastewater without any device (control), the XFiltra filter was the most successful device. This captured microfibres, reducing their release to wastewater by around $78 \%$. The Guppyfriend bag was the second most successful device, reducing microfibre release to wastewater by around 54\%; it appeared to mainly work by reducing microfibre shedding from the clothing during the washing cycle. Despite some potentially promising results it is important to recognise that fibres are also released when garments are worn in everyday use. Researchers and industry need continue to collaborate to better understand the best intervention points to reduce microfibre shedding, by considering both product design and fibre capture. 
Keywords: Microfibres; Washing Machines; Plastic Pollution; Microplastics;

Solutions

\section{$\underline{1.0 \text { Introduction }}$}

Textiles have a wide range of applications, including clothing, upholstery and carpeting, with global textile fibre production exceeding 106 million tons in 2018 (The Fiber Year, 2019); approximately 63\% of textile fibres produced are synthetic (e.g., polyester, nylon) (The Fiber Year, 2019). Other textile fibre materials include natural (e.g., cotton, wool) and semi-synthetic or regenerated fibres (e.g., rayon, acetate). While these types of fibres are produced from natural materials, such as wood pulp or cotton, natural and semi-synthetic fibres can be heavily modified with chemical treatments and additives (e.g., colourants, flame retardants) (Lacasse and Baumann, 2004; Xue et al., 2017). In this paper the term microfibre will refer exclusively to fibres (synthetic, semi-synthetic and natural) that are typically $<5 \mathrm{~mm}$.

It has been suggested that a large proportion of the microfibres found in the marine environment are released from textiles; with a key source being washing clothes (Belzagui et al., 2019; Cesa et al., 2020; De Falco et al., 2018; Napper and Thompson, 2016). On a global scale, Boucher and Friot, (2017) estimated that of all primary microplastics in the world's oceans, 35\% arise from laundry of synthetic textiles; an estimated 2 - 13 million tons per year globally (Boucher and Friot, 2017; Mishra et al., 2019). However, due to the lack of research on the release of natural and semi-synthetic fibres, this value is likely substantially underestimated. Microfibres can be released from clothing by mechanical stresses that fabrics undergo during the washing process in a washing machine (Belzagui et al., 2019; Cesa et al., 2020; De Falco et al., 2018; Napper and Thompson, 2016).

7 The first paper to highlight the importance of microfiber release form clothing was 68 that of Browne et al 2011 More recently, Napper and Thompson, (2016) estimated 
that a typical wash $(6 \mathrm{~kg})$ could produce over 700,000 microfibres. Since then, there has been further research focussing on microplastics from washing clothes using filters with fine mesh to capture the microfibres released ( $5 \mu \mathrm{m}$ mesh pore size in De Falco et al., (2018) compared to $25 \mu \mathrm{m}$ in Napper and Thompson, (2016)). As a consequence, it has recently been estimated that over 6,000,000 microfibres could be released from an average $6 \mathrm{~kg}$ wash (De Falco et al., 2018).

In addition to the pore size used to capture microfibers, release estimates can be influenced by differences in materials tested (whole garments vs. textile swatches; textile construction; material composition), load composition (mix loads; full loads; single garments), laundering conditions (temperature; detergent use; cycle time; water volume) and laundering methods (simulated laundering vs. household appliances; model; fibre enumeration and characterization) (Belzagui et al., 2019; Cesa et al., 2020; De Falco et al., 2018; Napper and Thompson, 2016). Currently, there is little scientific consensus on factors influencing release or release estimates across the field.

Microfibres released as a result of washing clothes, exit the washing machine via the waste effluent. Depending on the place of use, this effluent either passes directly into the environment or is sent to municipal wastewater treatment plants (WWTPs). In a WWTP, microplastic removal from water can be up to 96\% (Carr et al., 2016; Murphy et al., 2016) prior to the water being released to the environment.

During intense rainfall events, influent to the WWTP can exceed the treatment facilities' handling capacity resulting in the direct discharge of untreated wastewater into rivers, lakes or coastal areas. These events, even if occasional, may have a substantial impact on the total amount of microfibres released to natural environments (Galafassi et al., 2019). Even if microfibres are intercepted during wastewater treatment, the resultant sewage sludge is often returned to the land as a fertilizer, hence microfibres are still released to the environment (Corradini et al., 2019; Gies et al., 2018; Kirchmann et al., 2017). For example, it has been estimated that a secondary WWTP that serves a 650,000 population (Glasgow, UK) with a removal efficiency of $98.41 \%$ could release 65 million microplastic particles (including microfibres) every day (Murphy et al., 2016). A WWTP with a lower 
retention ability (84\%) and a greater population equivalent $(1,200,000)$ could discharge up to 160 million particles per day in its effluent (Magni et al., 2019). It has been reported that the majority of particles detected in WWTPs are microfibres (Gies et al., 2018; Gündoğdu et al., 2018; Leslie et al., 2017).

The number of microfibres entering into the marine environment from WWTP is likely to be substantial. Additionally, there are other sources of microfibres into the environment such as tumble drying (Pirc et al., 2016), the wearing of clothes (De Falco et al., 2020) and industrial emissions (Xu et al., 2018). As a consequence, microfibres are now found in aquatic habitats and organisms on a global scale (Avio et al., 2020; Nelms et al., 2019; Obbard et al., 2014; Saturno et al., 2020). Several recent studies revealed the presence of microfibres in various environments, including freshwater and marine surface waters and sediments, as well as terrestrial ecosystems (Ding et al., 2019; González-Pleiter et al., 2020; Liu et al., 2018; Luo et al., 2019; Lusher et al., 2015; Miller et al., 2017; Simon-Sánchez et al., 2019; Taylor et al., 2016; Woodall et al., 2014).

To mitigate microfibre release in laundry effluent, various devices have been designed to divert and capture released microfibres. These include devices aimed to go in the washing machine drum during a wash cycle and external filters fitted to the washing machine drainpipe to filter microfibres from outgoing effluent. Mcllwraith et al., (2019) previously compared the removal efficiency of one in-drum device, the Cora Ball, and one external washing machine filter, the Lint LUV-R. Based on weight, the study reported microfibre reductions into the wastewater by $5 \%$ and $80 \%$ for the Cora Ball and Lint LUV-R, respectively.

A range of other products are now available, or are being developed, that have the specific intent to reduce microfibre release. However, there is little data comparing efficacy among such devices. Given the accumulation of plastics in the environment has been associated with a lack of thorough consideration and evaluation of products at the design stage, it is therefore of key importance that any interventions should be appropriately evaluated. Therefore, the overall aim of this study was to 
examine which devices were the most effective at mitigating the release of

138 microfibers during a typical clothes wash. Efficiency in terms of reducing the release

139 of microfibers to waste water was also compared with control washes that had no

140 device present.

Our hypothesis assumed that devices would reduce microfibers entering the wastewater from clothes as a consequence of laundering. We chose to quantify the amount of microfibres by analysing the mass collected from the wastewater after washing three jumpers; i.e. microfibres released and that were unsuccessfully captured by the devices.

\subsection{Method}

\section{$\underline{2.1 \text { Materials }}$}

Three different synthetic fabric types were included in the washing trials to represent a typical mixed load $(1.3 \pm 0.2 \mathrm{~kg})$. These were medium sized jumpers, sourced from Primark (U.K.), made either of $100 \%$ polyester, $100 \%$ acrylic or $60 \%$ polyester / $40 \%$ cotton blend. Each load consisted of a whole garment from each fabric type. In order to identity each fabric type, microfibre samples from five replicates of each jumper type were analysed by FT-IR microscopy in transmission mode with a Hyperion 1000 microscope coupled to a Vertex 70 spectrometer (Bruker). Any spectra were recorded with 32 scans in the region of $4000-600 \mathrm{~cm}$. The spectra obtained were compared against a spectral database of synthetic polymers (BPAD polymer and synthetic fibres ATR). Napper and Thompson, (2016) had previously shown that garments had an initial peak of microfibre shedding in the first 1-4 washes and then a consistent microfibre shed after the fifth wash. Therefore, prior to data collection, any initial spike in microfibre loss from new clothes was reduced by washing each

168 fabric four times. 
172 The devices tested included three in-drum devices: the Guppyfriend washing bag

173 (Langbrett, Germany), a prototype Fourth Element washing bag (Fourth Element,

174 U.K.) and the Cora Ball (Cora Ball, VT, USA). Three external washing machine filters

175 were also tested, including: the Lint LUV-R (Environmental Enhancements, NS,

176 Canada), a prototype XFiltra (Xeros Technology Group, U.K.), and the PlanetCare

177 (PlanetCare Limited, U.K.) (Table 1). All devices were obtained in 2018; however, we

178 understand some manufactures (e.g PlanetCare and Fourth Element Washing Bag)

179 have been working on revised designs. Control washes using the same clothing but

180 without either an in-drum device or external filter were completed following the same

181 methodology. This determined how many fibres were released from the colthign in

182 the absence of any intervention device and allowed is to calculate microfibre capture

183 efficiency.

There were four replicates of each device and each was used in conjunction with an identical front-loading washing machine of $7 \mathrm{~kg}$ capacity (Hotpoint CarePlus WMAOD743P; $n=4$ ).. The mesh used in each device (minus Cora Ball which had no mesh) was visualised by scanning electron microscopy (JEOL, 7001F; Plymouth Electron Microscopy Centre) to assess the pore size.

Each device and controls were independently tested with four identical replicate mixed clothing loads coupled with four separate washing machines. Each mixed clothing load was washed 10 times, with data recorded after the $1^{\text {st }}, 5^{\text {th }}$ and $10^{\text {th }}$ wash (Fig. 1). The washing cycle setting was a 45 -minute synthetic wash at $30^{\circ} \mathrm{C}$ and 1000 R.P.M. This was chosen as a typical automatic programme chosen from the washing machine options (14 programmes available in total). The washing machines did not include weight measurement, so the volume of water used for each wash was consistent throughout (approximately $50 \mathrm{~L}$ of water). No detergent or conditioner was used as this would have left deposits affecting any weight change recorded. Additionally, all of the clothing was unwashed and new, so no other foreign contaminants would have affected the weight recorded (i.e. dirt). After washing the mixed loads, each replicate was tumble dried in a condenser dryer using an INDESIT IDC8T3 for 1 hour. 


\subsection{Analysis of Microfibres Captured/Released After Device Testing}

214

215 For each wash, the mass of microfibres that evaded capture were recorded from

216 each device. After each washing cycle, effluent together with any microfibres which

217 were not caught by the devices were collected in a storage tank and then pumped

218 into a $1 \mu \mathrm{m}$ filter cartridge (10", Sterner) which was stored in filter housing

219 (AQUAFILTER FHPR1-B1-AQ) (Fig. 2). Aluminium bungs were custom made to

220 block the bottom end of the cartridges; subsequently, the wastewater was pushed

221 through the cartridge leaving any microfibres trapped in its mesh. Cartridges were

222 weighed before and after each wash cycle. The dry weight was recorded for each

223 cartridge after being dried at $30^{\circ} \mathrm{C}$ to a constant weight and then weighed by a

224 Cubis $₫$ precision balance (Sartorius). The cartridges were wrapped in two layers of

225 foil during the drying process to stop microfibre loss or addition of contamination.

227 The weight of microfibres successfully captured as well as subjective observations

228 on the ease of use of the devices were recorded; this was completed to understand

229 the mechanism of each device, rather than just efficiency testing. For devices where

230 the consumer was expected to visually inspect and then remove the microfibres

231 (Cora Ball, Guppyfriend and Fourth Element washing bag) a timed 5-minute

232 inspection period was used to ensure a sensible and consistent consumer removal

233 effort scenario. This inspection period also provided substantially enough time to

234 remove the majority of collected microfibre mass. This was completed as a

235 consumer would (i.e. without gloves or forceps) and by one person, to reduce

236 variability among individuals. For the PlanetCare filters, the microfibres could not be

237 removed from the device due to being collected into a sealed filter. These filters are 
238 intended to be returned to PlanetCare for recycling. Therefore, the dry weight

239 change of the PlanetCare filter itself was recorded.

2.4. Quality Assurance and Quality Control

During testing and analysis, all steps were conducted in a regularly cleaned laboratory with controlled access. Care was taken to ensure any potential sources of airborne contamination were minimised (Woodall et al., 2015).. Additionally, all analytical equipment was shielded to mitigate any exposure or contamination throughout the washing and drying process. During analysis (e.g. weighing or sample preparation), procedural blanks were conducted after every $5^{\text {th }}$ sample and confirmed microplastic contamination was minimal with an average of $2 \pm 1$ microfibers filter ${ }^{-1}$. This was negligible to the amount of fibres being captured during a wash cycle. After each washing machine cycle which involved mixed clothing loads, cross contamination was minimized between washes, by running the washingmachine at $30{ }^{\circ} \mathrm{C}, 1000$ R.P.M for 45 min with no fabric present.

\subsection{Statistical Analysis}

Normality of the data was confirmed by using QQ plots to examine distribution. Differences between the six devices in terms of the mass of microfibres captured and released were then analysed using 2-way ANOVAs with device and time point as fixed factors. Examination of residuals of the fitted modules indicated the need for transformation (logarithm transformation) of both datasets; residuals were unbiased and homoscedastic after transformation. Post-hoc Tukey tests were used to identify statistically significant differences between devices. Standard error of the mean was used for all analysis. 
274 Washing a mixed load of clothes without any device (control testing), resulted in an average of $0.44 \mathrm{~g} \pm 0.04 \mathrm{~g}$ (mean $+\mathrm{S}$.E) of microfibres being released into the wastewater effluent per wash (Fig. 3A). This estimate (which is assumed to represent $0 \%$ success in terms of microfibre shedding mitigation or capture) was then compared against the mass of microfibres collected from wastewater effluent with each device. Higher efficiency (\%) equates to a more successful device. When comparing between devices and control, the devices ranged between $21-78 \%$ efficiency. XFiltra was the most successful device, reducing the number of microfibres being released into the wastewater by $78 \pm 5 \%$. The Guppyfriend washing bag was the second most successful device at $54 \pm 14 \%$. The Cora Ball was the third most successful at $31 \pm 8 \%$. The Lint LUV-R and PlanetCare had similar results at $29 \pm 15 \%$ and $25 \pm 20 \%$, respectively. The Fourth Element washing bag was the least effective at $21 \pm 9 \%$ (Fig. 3A).

There were significant differences in the mass of microfibres released into wastewater across devices (2-way ANOVA; $p=<0.008$ ); these differences were consistent across the three timepoints (Table 2). At the 0.05 level, the Guppyfriend washing bag and XFiltra were the only devices to release significantly less microfibres compared to controls (no device). There were no significant differences between microfibre release by in-drum devices (Fig. 3A). XFiltra also released significantly less microfibres than the Cora Ball, Fourth Element washing bags, Lint LUV-R and Planetcare.

There was also a significant difference in the mass of microfibres successfully captured by each device type (2-way ANOVA; $p=<0.000$ ) (Fig. 3B). There was no significant difference, at the 0.05 level, between Cora Ball, Guppyfriend and Fourth Element washing bags (Fig. 3B). Trying to manually remove the microfibres from devices added to the drum (Cora Ball, Guppyfriend and Fourth Element washing bags) was time consuming as there was a large surface area to analyse and little mass typically collected. With the Guppyfriend washing bag, microfibres typically accumulated in the hem of material. However, for the external filters (XFiltra and Lint LUV-R), microfibres would typically accumulate in a localised area. PlanetCare 
captured microfibres were irretrievable due to the devices design; these filters are intended to be returned to PlanetCare for recycling.

Scanning electron images were obtained to assess the pore size of the mesh used in each device (apart from the Cora Ball, which contained no mesh) (Fig. 4). The largest pore size was the Lint LUV-R, which had 2 pore sizes: $285 \mu \mathrm{m}$ and 175 $\mu \mathrm{m}$. PlanetCare had the second largest pore size of $200 \mu \mathrm{m}$. XFiltra had a pore size of $60 \mu \mathrm{m}$. The two bag devices (Guppyfriend and Forth Element washing bag) had the smallest pore size, of $50 \mu \mathrm{m}$.

\subsection{Discussion}

The XFiltra prototype device was the most successful device, capturing on average $78 \%$ of the microfibres per wash. It is possible that this device was more successful firstly because it had the finest mesh pore size $(60 \mu \mathrm{m})$ compared to the other filters (PlanetCare \& Lint LUV-R) which had pore sizes $>175 \mu \mathrm{m}$, and secondarily, because it was the only 'active device', in that it used a motor powered centrifugal separator requiring an external electrical supply to facilitate the flow of the waste water through the filtration mesh. There was also a large variation in efficiency between the Guppyfriend and Fourth Element washing bags; $54 \%$ and $21 \%$, respectively. Even though each bag device had similar mesh pore size $(50 \mu \mathrm{m})$, their shape and design were different which could account for differences in efficiency.

Additionally, our results found that there was a significant difference in the mass of microfibres captured by the devices. Devices directly placed into the washing machine drum (Cora Ball, Guppyfriend and Fourth Element washing bags) were all less successful at capturing microfibres than the filters, but were still found to reduce microfibre emissions into the wastewater by $21-54 \%$. This effect seems to have resulted from reduced microfibre shedding by garments during the washing cycle due to the design of these devices.

Previous research has demonstrated that the Cora Ball and the Lint LUV-R reduced the weight of microfibres released after a washing cycle by $5 \%$ and $80 \%$, 
340 respectively (Mcllwraith et al., 2019). However, we report that the Lint LUV-R to be 341 less successful at $29 \%$, and the Cora Ball at $31 \%$. One possible explanation for the

342 differences between studies could be because Mcllwraith et al., (2019) did not focus 343 on microfibres smaller than $10 \mu \mathrm{m}$, whereas this study had a lower limit of $1 \mu \mathrm{m}$.

344 Additionally, there are differences in study design. Mcllwraith et al. (2019) used

$345100 \%$ polyester fleece blankets, which have been reported to have high shedding 346 rates (Browne et al., 2011; Pirc et al., 2016; Sillanpää and Sainio, 2017). Their

347 research also used a top loading machine which is suspected to shed more

348 microfibres from clothing/fabric compared to a frontloading machine (Hartline et al., 349 2016).

351 Despite removing $21-78 \%$ of outgoing microfibres, the six devices tested in the present study still released $0.10-0.35 \mathrm{~g}$ of microfibres per wash. As such they do not offer a complete solution and alternative measures will likely still need to be taken to address this issue. A combination of in-drum and external filter technologies used together may cause less shedding and increased microfibre capture, whilst also reducing the need to clean the filter as frequently.

Additionally, reducing shedding through changes in fabric design could be a more overarching mitigation strategy, as this is likely to help reduce emissions during all use phases: wearing, washing and tumble drying (De Falco et al., 2020; Napper and Thompson, 2016; Pirc et al., 2016). De Falco et al., (2020) estimated the quantity of microfibres released into the air directly as a consequence of wearing clothes. Their research found that 400 fibres gram $^{-1}$ of fabric could be shed by items of clothing during just 20 minutes of normal activity. Due to this, it is anticipated that atmospheric deposition of microplastics, especially through the wearing of clothes, is a substantial pathway into the environment. Microplastics are potentially transported by wind, because of their small size and low density, from their original source (Bergmann et al., 2019).

Other measures can be put in place to minimise microfibres shed in the washing cycle. Only washing your clothes when required is a simple way to minimise microfibre shedding. Research has also indicated that delicate wash cycles release 
more microfibres per wash than a lower water-volume standard wash; showing that simply reducing the water-volume-to-fabric ratio could also have an effect in reducing the amount of microfibres generated (Kelly et al., 2019). Therefore, an effective strategy would be using a combination of modified fabric design together with less aggressive washing cycles and adding washing machine filters/in-drum devices.

More research is needed to establish how regularly consumers would actually clean the devices (we considered a 5-minute clean to be a reasonably generous amount of time). It is unclear what consumers would do with any microfibres removed; e.g. dispose to landfill or wash them down the sink unintentionally to clean the device. Clear labelling and instructions should be in place to ensure the proper disposal of microfibres. There are further limitations to the widespread implementation of these devices. For the in-drum devices, research should analyse whether garments being laundered receive the same quality of cleaning. Due to the size of the washing bags, the consumer is also limited in the number of clothes able to be laundered, so more washes may be required. Additionally, the external washing machine filters will require potential space for installation in washing machines. All devices vary in price and are currently assumed to be purchased by the consumer, although there is the potential for washing machine manufactures to incorporate filters internally in production.

Other mitigation strategies that have been promoted include improvements to WWTPs and a switch from synthetic to natural textiles. However, these solutions are more unrealistic. WWTP microplastic removal can already be up to 96\% (Carr et al., 2016; Murphy et al., 2016) prior to the water being released to the environment. Upgrading WWTP with more efficient filtering systems could be expensive or potentially not even possible with the system already in place (Conley et al., 2019). Furthermore, replacing synthetic textiles with natural counterparts would typically be more expensive and the impact of non-synthetic microfibres accumulating in the environment is also currently unknown (Dris et al., 2017). because of inadequate consideration at the industrial design stage of the environmental consequences associated with production, use and disposal. Going 
408 forward it is imperative we learn from these mistakes. From the perspective of

409 interventions to tackle current issues with laundering, this needs to be done in terms

410 of their efficacy in addressing the particular issue and potential unintended

411 environmental consequences. From an environmental perspective we can no longer

412 afford to produce devices and products in the hope they will be not be harmful, rather

413 we must rigorously assess performance, prior to release. Industries will continue to

414 develop solutions aimed to stem the flow of or capture plastic getting into the

415 environment. However, it is essential that any proposed solutions are fully tested for

416 their efficiency and evaluated to understand their potential benefit.

417

$418 \underline{5.0 \text { Conclusion }}$

419

420 There is now considerable agreement and consensus about the issue of plastic

421 waste and pollution. However, some of the key challenges now lie, not just in environmental science to help understand the problem, but robust evidence to inform appropriate solutions. With growing concern about the accumulation of plastic and microplastic (including concern about microfibre pollution) devices are being developed with the intent to reduce the release of microfibres to the environment. These solutions vary in their approach, such as providing consumer ease or being the most effective. They also vary in market readiness. Our study has shown they vary in their ability to address the issue of microfibre contamination. XFiltra and the Guppyfriend washing bag significantly reduced the number of microfibres released into the wastewater compared to no device being present. In order to help minimise some of the avoidable environmental challenges that we currently face, it is essential that technological advance is coupled, at the design stage, to appropriate environmental science, in order to minimise unintended environmental consequences. 
Avio, C.G., Pittura, L., d'Errico, G., Abel, S., Amorello, S., Marino, G., Gorbi, S., Regoli, F., 2020. Distribution and characterization of microplastic particles and textile microfibers in Adriatic food webs: General insights for biomonitoring strategies. Environ. Pollut. 258, 113766. doi:10.1016/j.envpol.2019.113766 Belzagui, F., Crespi, M., Álvarez, A., Gutiérrez-Bouzán, C., Vilaseca, M., 2019. Microplastics' emissions: Microfibers' detachment from textile garments. Environ. Pollut. 248, 1028-1035. doi:10.1016/j.envpol.2019.02.059

Bergmann, M., Mützel, S., Primpke, S., Tekman, M.B., Trachsel, J., Gerdts, G., 2019. White and wonderful? Microplastics prevail in snow from the Alps to the Arctic. Sci. Adv. 5, eaax1157. doi:10.1126/sciadv.aax1157

Boucher, J., Friot, D., 2017. Primary Microplastics in the Oceans: a Global Evaluation of Sources. Gland, Switzerland.

Browne, M.A., Crump, P., Niven, S.J., Teuten, E., Tonkin, A., Galloway, T., Thompson, R., 2011. Accumulation of microplastic on shorelines woldwide: Sources and sinks. Environ. Sci. Technol. 45, 9175-9179. doi:10.1021/es201811s

Carr, S.A., Liu, J., Tesoro, A.G., 2016. Transport and fate of microplastic particles in wastewater treatment plants. Water Res. 91, 174-182. doi:10.1016/j.watres.2016.01.002

Cesa, F.S., Turra, A., Checon, H.H., Leonardi, B., Baruque-Ramos, J., 2020. Laundering and textile parameters influence fibers release in household washings. Environ. Pollut. 257, 113553. doi:10.1016/j.envpol.2019.113553

Conley, K., Clum, A., Deepe, J., Lane, H., Beckingham, B., 2019. Wastewater treatment plants as a source of microplastics to an urban estuary: Removal efficiencies and loading per capita over one year. Water Res. X 3, 100030. doi:10.1016/j.wroa.2019.100030

Corradini, F., Meza, P., Eguiluz, R., Casado, F., Huerta-Lwanga, E., Geissen, V., 2019. Evidence of microplastic accumulation in agricultural soils from sewage sludge disposal. Sci. Total Environ. 671, 411-420. doi:10.1016/J.SCITOTENV.2019.03.368

De Falco, F., Cocca, M., Avella, M., Thompson, R.C., 2020. Microfibre release to water, via laundering, and to air, via everyday use: a comparison between polyester clothing with differing textile parameters. Environ. Sci. Technol. 54, 3288-3296. doi:10.1021/acs.est.9b06892 
De Falco, F., Gullo, M.P., Gentile, G., Di Pace, E., Cocca, M., Gelabert, L., BroutaAgnésa, M., Rovira, A., Escudero, R., Villalba, R., Mossotti, R., Montarsolo, A., Gavignano, S., Tonin, C., Avella, M., 2018. Evaluation of microplastic release caused by textile washing processes of synthetic fabrics. Environ. Pollut. 236, 916-925. doi:10.1016/J.ENVPOL.2017.10.057

Ding, L., Mao, R. fan, Guo, X., Yang, X., Zhang, Q., Yang, C., 2019. Microplastics in surface waters and sediments of the Wei River, in the northwest of China. Sci. Total Environ. 667, 427-434. doi:10.1016/J.SCITOTENV.2019.02.332

Dris, R., Gasperi, J., Mirande, C., Mandin, C., Guerrouache, M., Langlois, V., Tassin, B., 2017. A first overview of textile fibers, including microplastics, in indoor and outdoor environments. Environ. Pollut. 221, 453-458. doi:10.1016/j.envpol.2016.12.013

Galafassi, S., Nizzetto, L., Volta, P., 2019. Plastic sources: A survey across scientific and grey literature for their inventory and relative contribution to microplastics pollution in natural environments, with an emphasis on surface water. Sci. Total Environ. 693, 133499. doi:10.1016/j.scitotenv.2019.07.305

Gies, E.A., LeNoble, J.L., Noël, M., Etemadifar, A., Bishay, F., Hall, E.R., Ross, P.S., 2018. Retention of microplastics in a major secondary wastewater treatment plant in Vancouver, Canada. Mar. Pollut. Bull. 133, 553-561. doi:10.1016/j.marpolbul.2018.06.006

González-Pleiter, M., Velázquez, D., Edo, C., Carretero, O., Gago, J., Barón-Sola, Á., Hernández, L.E., Yousef, I., Quesada, A., Leganés, F., Rosal, R., Fernández-Piñas, F., 2020. Fibers spreading worldwide: Microplastics and other anthropogenic litter in an Arctic freshwater lake. Sci. Total Environ. 722, 137904. doi:10.1016/j.scitotenv.2020.137904

Gündoğdu, S., Çevik, C., Güzel, E., Kilercioğlu, S., 2018. Microplastics in municipal wastewater treatment plants in Turkey: a comparison of the influent and secondary effluent concentrations. Environ. Monit. Assess. 190, 626. doi:10.1007/s10661-018-7010-y

Hartline, N.L., Bruce, N.J., Karba, S.N., Ruff, E.O., Sonar, S.U., Holden, P.A., 2016. Microfiber Masses Recovered from Conventional Machine Washing of New or Aged Garments. Environ. Sci. Technol. 50, 11532-11538. doi:10.1021/acs.est.6b03045 Kelly, M.R., Lant, N.J., Kurr, M., Burgess, J.G., 2019. Importance of Water-Volume 

11735-11744. doi:10.1021/acs.est.9b03022

Kirchmann, H., Börjesson, G., Kätterer, T., Cohen, Y., 2017. From agricultural use of sewage sludge to nutrient extraction: A soil science outlook. Ambio 46, 143154. doi:10.1007/s13280-016-0816-3

Lacasse, K., Baumann, W., 2004. Textile Chemicals - Environmental Data and Facts. Springer, Berlin, Germany.

Leslie, H.A., Brandsma, S.H., van Velzen, M.J.M., Vethaak, A.D., 2017.

Microplastics en route: Field measurements in the Dutch river delta and Amsterdam canals, wastewater treatment plants, North Sea sediments and biota. Environ. Int. 101, 133-142. doi:10.1016/j.envint.2017.01.018

Liu, M., Lu, S., Song, Y., Lei, L., Hu, J., Lv, W., Zhou, W., Cao, C., Shi, H., Yang, X., He, D., 2018. Microplastic and mesoplastic pollution in farmland soils in suburbs of Shanghai, China. Environ. Pollut. 242, 855-862. doi:10.1016/J.ENVPOL.2018.07.051

Luo, W., Su, L., Craig, N.J., Du, F., Wu, C., Shi, H., 2019. Comparison of microplastic pollution in different water bodies from urban creeks to coastal waters. Environ. Pollut. 246, 174-182. doi:10.1016/J.ENVPOL.2018.11.081

Lusher, A.L., Tirelli, V., O'Connor, I., Officer, R., Halsband, C., Galloway, T.S., 2015. Microplastics in Arctic polar waters: the first reported values of particles in surface and sub-surface samples. Sci. Rep. 5, 14947. doi:10.1038/srep14947

Magni, S., Binelli, A., Pittura, L., Avio, C.G., Della Torre, C., Parenti, C.C., Gorbi, S., Regoli, F., 2019. The fate of microplastics in an Italian Wastewater Treatment Plant. Sci. Total Environ. 652, 602-610. doi:10.1016/J.SCITOTENV.2018.10.269

Mcllwraith, H.K., Lin, J., Erdle, L.M., Mallos, N., Diamond, M.L., Rochman, C.M., 2019. Capturing microfibers - marketed technologies reduce microfiber emissions from washing machines. Mar. Pollut. Bull. 139, 40-45. doi:10.1016/J.MARPOLBUL.2018.12.012

Miller, R.Z., Watts, A.J.R., Winslow, B.O., Galloway, T.S., Barrows, A.P.W., 2017. Mountains to the sea: River study of plastic and non-plastic microfiber pollution in the northeast USA. Mar. Pollut. Bull. 124, 245-251. doi:10.1016/J.MARPOLBUL.2017.07.028 Mishra, S., Rath, C. charan, Das, A.P., 2019. Marine microfiber pollution: A review 
on present status and future challenges. Mar. Pollut. Bull. doi:10.1016/j.marpolbul.2019.01.039

Murphy, F., Ewins, C., Carbonnier, F., Quinn, B., 2016. Wastewater Treatment Works (WwTW) as a Source of Microplastics in the Aquatic Environment. Environ. Sci. Technol. 50, 5800-5808. doi:10.1021/acs.est.5b05416

Napper, I.E.I., Thompson, R.C., 2016. Release of synthetic microplastic plastic fibres from domestic washing machines: Effects of fabric type and washing conditions. Mar. Pollut. Bull. 112, 39-45. doi:10.1016/j.marpolbul.2016.09.025

Nelms, S.E., Barnett, J., Brownlow, A., Davison, N.J., Deaville, R., Galloway, T.S., Lindeque, P.K., Santillo, D., Godley, B.J., 2019. Microplastics in marine mammals stranded around the British coast: ubiquitous but transitory? Sci. Rep. 9, 1075. doi:10.1038/s41598-018-37428-3

Obbard, R.W., Sadri, S., Wong, Y.Q., Khitun, A.A., Baker, I., Thompson, R.C., 2014. Global warming releases microplastic legacy frozen in Arctic Sea ice. Earth's Futur. 2, 315-320. doi:10.1002/2014EF000240.Abstract

Pirc, U., Vidmar, M., Mozer, A., Kržan, A., 2016. Emissions of microplastic fibers from microfiber fleece during domestic washing. Environ. Sci. Pollut. Res. 23, 22206-22211. doi:10.1007/s11356-016-7703-0

Saturno, J., Liboiron, M., Ammendolia, J., Healey, N., Earles, E., Duman, N., Schoot, I., Morris, T., Favaro, B., 2020. Occurrence of plastics ingested by Atlantic cod (Gadus morhua) destined for human consumption (Fogo Island, Newfoundland and Labrador). Mar. Pollut. Bull. 153, 110993.

doi:10.1016/j.marpolbul.2020.110993

Sillanpää, M., Sainio, P., 2017. Release of polyester and cotton fibers from textiles in machine washings. Environ. Sci. Pollut. Res. 24, 19313-19321. doi:10.1007/s11356-017-9621-1

Simon-Sánchez, L., Grelaud, M., Garcia-Orellana, J., Ziveri, P., 2019. River Deltas as hotspots of microplastic accumulation: The case study of the Ebro River (NW Mediterranean). Sci. Total Environ. 687, 1186-1196.

doi:10.1016/J.SCITOTENV.2019.06.168

Taylor, M.L., Gwinnett, C., Robinson, L.F., Woodall, L.C., 2016. Plastic microfibre ingestion by deep-sea organisms. Sci. Rep. 6, 33997. doi:10.1038/srep33997

The Fiber Year, 2019. The Fiber Year 2019; World Survey on Textiles \& Nonwovens. Frankfurt, Germany. 
578 Woodall, L.C., Sanchez-Vidal, A., Canals, M., Paterson, G.L.J.J., Coppock, R.,

579 Sleight, V., Calafat, A., Rogers, A.D., Narayanaswamy, B.E., Thompson, R.C.,

580 2014. The deep sea is a major sink for microplastic debris. R. Soc. Open Sci. 1,

581 140317-140317. doi:10.1098/rsos.140317

582 Xu, C.K., Cheng, H., Liao, Z.J., 2018. Towards sustainable growth in the textile

583 industry: A case study of environmental policy in China. Polish J. Environ. Stud.

$584 \quad 27,2325-2336$. doi:10.15244/pjoes/79720

585 Xue, J., Liu, W., Kannan, K., 2017. Bisphenols, Benzophenones, and Bisphenol A

586 Diglycidyl Ethers in Textiles and Infant Clothing. Environ. Sci. Technol. 51,

$587 \quad$ 5279-5286. doi:10.1021/acs.est.7b00701

588

589

590

591

592 